\title{
Assessment of the pathway of apoptosis involving PAR-4, DAXX and ZIPK proteins in CLL patients and its relationship with the principal prognostic factors
}

\author{
Agnieszka Bojarska-Junak ${ }^{1 *}$, Małgorzata Sieklucka1, 2*, Iwona Hus ${ }^{2}$, \\ Ewa Wąsik-Szczepanek², Maria Luiza Kusz², Agata Surdacka1, \\ Sylwia Chocholska², Anna Dmoszyńska², Jacek Roliński ${ }^{1}$ \\ ${ }^{1}$ Department of Clinical Immunology, Medical University of Lublin, Poland \\ ${ }^{2}$ Department of Hematooncology and Bone Marrow Transplantation, Medical University of Lublin, Poland \\ *Equal contribution of both authors
}

\begin{abstract}
Par-4 (prostate apoptosis response-4) protein was originally found upregulated in prostate tumor cells undergoing apoptosis. Then it was further identified as a proapoptotic protein upregulated both in normal and leukemic lymphocytes. The aim of our study was to assess PAR-4 protein expression in the B cells of CLL patients and to examine its relationship with the expression of other proteins involved in the apoptosis process, such as DAXX, ZIPK and BCL-2. We found a positive relationship between PAR-4 and BCL-2 protein expression. Additionally, there was a positive correlation between PAR-4 and both DAXX and ZIPK protein expression. The results of our research were also analyzed in association with the principal CLL prognostic factors. There was a positive correlation between the expression of PAR-4 protein and the lactate dehydrogenase $(\mathrm{LDH})$ serum concentration $(\mathrm{p}<0.005)$. The expression of PAR-4 protein in B cells correlated positively with the percentage of CD38 $8^{+}$cells $(\mathrm{p}<0.05)$, as well as with $\mathrm{CD} 38^{+} / \mathrm{ZAP}-70^{+}$cells $(\mathrm{p}<0.05)$. Moreover, we found a close relationship between LPL protein expression or LPL/ADAM29 MFI ratio and PAR-4 protein expression. Our results confirm the significance of apoptosis deregulation in CLL, and suggest a possible relationship between PAR-4 expression and the clinical course of the disease. This however requires further investigation. (Folia Histochemica et Cytobiologica 2011; Vol. 49, No. 1, pp. 98-103)
\end{abstract}

Key words: PAR-4, DAXX, ZIPK, BCL-2, CD38, apoptosis, chronic lymphocytic leukemia

\section{Introduction}

Prostate apoptosis response-4 (PAR-4) protein, the product of the proapoptotic gene PAR-4/PAWR (PRKC, apoptosis, WT1, regulator), was originally identified in prostate cancer cells that were induced to undergo apoptosis following androgen withdrawal

Correspondence address: A. Bojarska-Junak, Department of Clinical Immunology, Medical University of Lublin,

Chodzki Str. 4a, 20-093 Lublin, Poland;

tel.: (+ 48 81) 75648 40; fax: (+ 48 81) 75648 40;

e-mail: agnieszkajunak@poczta.onet.pl
[1-3]. It has been observed increased in rat prostate cells after castration $[2,4]$. PAR-4 is a leucine zipper domain protein of $\sim 38 \mathrm{kDa}$ that has been subsequently found widely expressed in various normal and malignant cell types and tissues [5-8]. It contains a death domain that is necessary to sensitize cells to apoptosis [7]. PAR-4 protein has been suggested as promoting apoptosis in various cell types in response to a variety of stimuli, such as chemotherapy, UV-radiation or elevation of intracellular calcium concentration $[4,9]$. Furthermore, it has been found that PAR-4 overexpression in nonhematopoietic cells results in inducing apoptosis by 
downregulating BCL-2 protein level, and it is known that BCL-2 family members are among the key regulators of the apoptotic process $[9,10]$. Moreover, PAR-4 promotes the Fas apoptosis pathway and parallel NF- $\kappa$ B inhibition [11].

In subsequent studies, PAR-4 has been identified upregulated both in normal and leukemic lymphocytes [9]. In lymphatic cells, increased levels of PAR-4 protein were followed by BCL-2 protein downregulation and cleavage of poly(ADP-ribose) polymerase (PARP) [10]. PAR-4 has been suggested as playing a role both in extrinsic and intrinsic apoptotic pathway and it co-operates with other proapoptotic proteins, including the nuclear ZIP kinase (ZIPK) and DAXX (death-associated protein) [12, 13].

ZIPK was first identified as a serine/threonine kinase that binds ATF4, a member of the activating transcription factor/cyclic AMP-responsive elementbinding protein $(\mathrm{ATF} / \mathrm{CREB})$ family of transcription factors [14]. ZIPK is a proapoptotic protein kinase initiating a nuclear apoptotic pathway in collaboration with PAR-4 and DAXX proteins [13].

DAXX is a multi-functional protein suggested as playing a role in several important apoptotic pathways. Originally, DAXX was identified as a proapoptotic Fas-binding protein able to enhance the extrinsic apoptosis pathway through Jun N-terminal kinase activation [15].

There is little data concerning the PAR-4 pathway in chronic lymphocytic leukemia (CLL). The aim of our study was to assess this protein expression in the B cells of CLL patients and examine the relationship between PAR-4 and other proteins involved in apoptosis, such as BCL-2, ZIP kinase and DAXX.

\section{Material and methods}

\section{Patients and samples}

Peripheral blood (PB) and bone marrow (BM) specimens were obtained from 62 untreated CLL patients diagnosed between September 2005 and December 2009 (32 men and 30 women). The median age of patients was 66 years (range 32 to 87). CLL diagnosis was based on a clinical examination, morphological and immunological criteria [16]. At the time of diagnosis, patients were staged according to the Rai staging system [17] as follows: stage 0 (21 cases), stage 1 (19 cases), stage 2 (12 cases), stage 3 (three cases) and stage 4 (seven cases). The patient cohort was divided into three groups: patients with Rai stage 0 (21 cases), stage 1-2 (31 cases) and stage 3-4 (ten cases). PB samples were collected into heparinized tubes and immediately processed. The study was approved by the Local Ethics Committee.

\section{Intracellular analysis of PAR-4, DAXX and ZIP kinase}

Intracellular PAR-4 staining was performed with anti-PAR mouse $\operatorname{IgG}_{2 \mathrm{a}}$ antibody solution from Santa Cruz Biotechnology labeled using the Zenon ${ }^{\mathrm{TM}}$ Alexa Fluor 488 Mouse $\operatorname{IgG}_{2 \mathrm{a}}$ Labeling Kit (Molecular Probes) according to the manufacturer's instructions. Intracellular DAXX staining was performed with anti-DAXX rabbit monoclonal antibody from EPITOM -ICS. Intracellular ZIP kinase analysis was performed with anti-ZIPK rabbit monoclonal antibody (Abcam). Anti-DAXX and anti-ZIPK antibodies solution were labeled using the Zenon ${ }^{\mathrm{TM}}$ Alexa Fluor 488 Rabbit Labeling Kit (Molecular Probes).

For intracellular detection of PAR-4, DAXX and ZIPK, PBMC were stained with monoclonal antibodies against cell surface markers, i.e. CD19 PE (20 minutes at RT). Following membrane staining, fixation/permeabilization procedures were performed (IntraPrep Kit, Immunotech). Cells were then incubated with anti-PAR-4, anti-DAXX or anti-ZIPK antibodies labeled by Zenon ${ }^{\mathrm{TM}}$ Alexa Fluor 488 Labeling Kits or with appropriate isotypic control for 20 minutes at room temperature.

In this study, levels of PAR-4, DAXX and ZIPK expression, indicated by mean fluorescence intensity (MFI), were analyzed.

\section{Intracellular analysis of $B C L-2$}

Intracellular BCL-2 protein analysis was performed with FITC conjugated anti-BCL-2 mouse monoclonal antibody (DAKO). PBMC were stained with monoclonal antibodies against cell surface markers, i.e. CD19 PE. Following membrane staining, fixation/ /permeabilization procedures were performed (IntraPrep Kit). Cells were then incubated with anti-BCL-2 antibody or appropriate isotypic control for 20 minutes at room temperature. The level of BCL-2 protein expression, indicated by MFI, was analyzed.

\section{Analysis of ZAP-70 expression in CLL cells}

All PB samples were stained for ZAP-70 protein expression. We used a previously described method for flow cytometric examination of ZAP-70 protein expression $[18,19]$. A cut-off point for ZAP-70 positivity in leukemic cells was $\geq 20 \%$.

\section{Detection of CD38 expression}

Flow cytometry analysis of CD38 antigen expression was performed on fresh $\mathrm{PB}$ samples, as described previously [19]. CLL cells were considered CD38-posi- 
tive when $\geq 20 \%$ of them expressed the membrane antigen.

\section{Analysis of LPL and ADAM29 expression in CLL cells}

Peripheral blood samples from 100 CLL patients were stained for LPL and ADAM29 expression. We used a previously described method for flow cytometric examination of LPL and ADAM29 expression [20]. The expression of ADAM29 and LPL is described as the mean fluorescence intensity (MFI).

\section{Flow cytometry analysis}

Samples were analyzed by two- and three-color flow cytometry using the Becton Dickinson FACS-Calibur instrument. Five data parameters were acquired and stored, i.e. linear forward and side scatter (FSC, SSC), green-fluorescence (FL-1), orange-fluorescence (FL-2) and red-fluorescence (FL-3). For each analysis, 10,000 events were acquired and analyzed using CellQuest software. An acquisition gate was established based on FSC and SSC that excluded dead cells and debris. Isotype-matched antibody was used to verify staining specificity and as a guide for setting markers used to delineate positive and negative populations.

\section{Statistical analysis}

Differences between two groups were assessed using the U Mann-Whitney test. The Spearman rank correlation coefficient was used in correlation tests. We used Statistica 7.0 PL software for all statistical procedures. Differences were considered statistically with a $p$ value $\leq 0.05$

\section{Results}

We found a positive relationship between PAR-4 and BCL-2 protein expression $(\mathrm{R}=0.7 ; \mathrm{p}<0.000001)$.

Additionally, there was a positive correlation between PAR-4 and both DAXX and ZIPK protein expression $(\mathrm{R}=0.6 ; \mathrm{p}<0.00005$ and $\mathrm{R}=0.5 ; \mathrm{p}<$ $<0.005$, respectively). Moreover, there was a positive correlation between DAXX and ZIPK protein expression $(\mathrm{R}=0.6 ; \mathrm{p}<0.0005)$ (Figures $1 \mathrm{~A}-\mathrm{D})$.

The results of our research were analyzed in association with the principal CLL prognostic factors. There was a positive correlation between the expression of PAR-4 protein and the lactate dehydrogenase (LDH) serum concentration $(\mathrm{R}=0.4 ; \mathrm{p}<0.005)$. However, we did not observe significant correlations between the PAR-4 protein expression and such prognostic factors
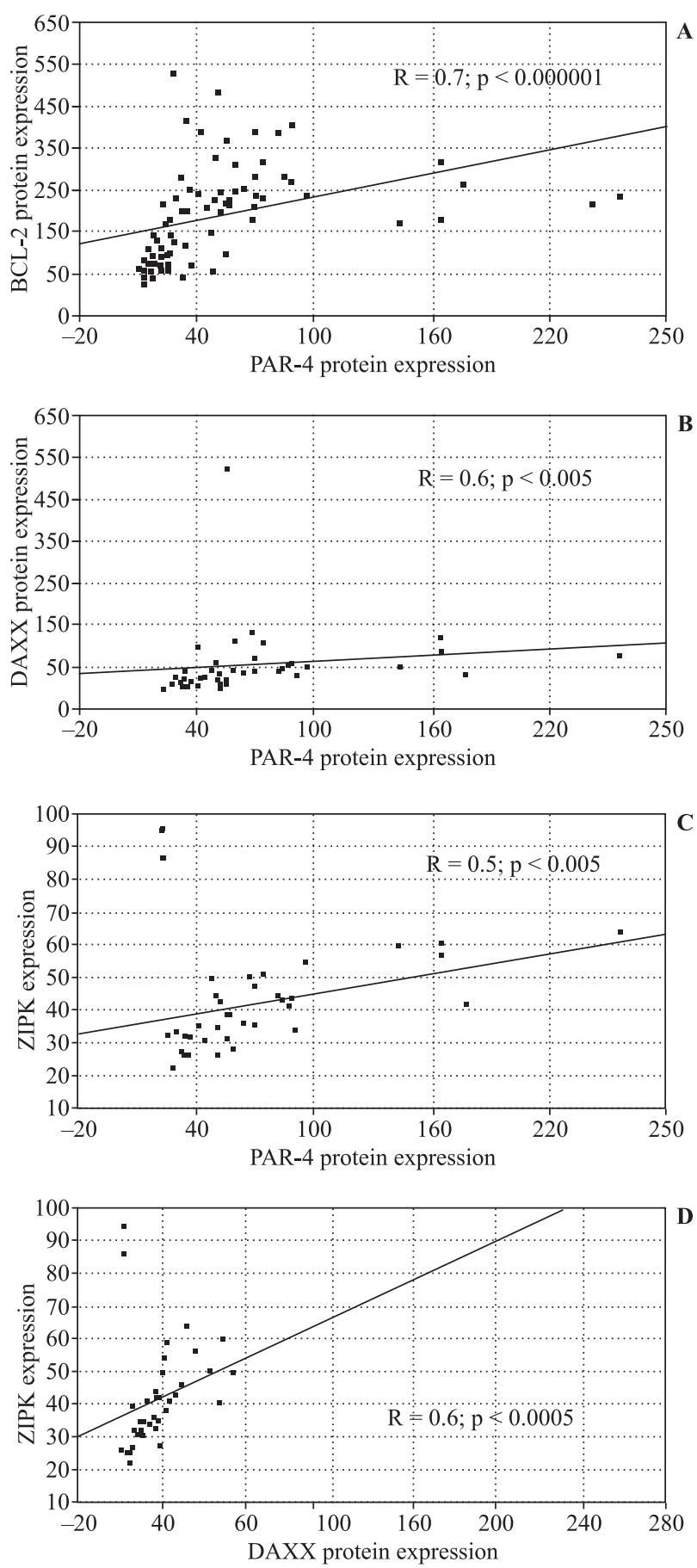

Figure 1. Relationship between PAR-4 and BCL-2, DAXX and ZIPK protein expression. MFI - mean fluorescence intensity

as leukocytosis and lymphocytosis, hemoglobin level, platelet count or $\beta_{2}$-microglobulin level.

The expression of PAR-4 protein in B cells correlated positively with the percentage of $\mathrm{CD} 38^{+}$cells $(\mathrm{R}=0.2 ; \mathrm{p}<0.05)$. We observed a significantly higher expression of PAR- 4 protein in $\mathrm{CD}_{3} 8^{+}$patients (median: 62.84 MFI) than in CD38- patients (median: $42.22 \mathrm{MFI})(\mathrm{p}<0.05)($ Figure $2 \mathrm{~A})$, as well as in 

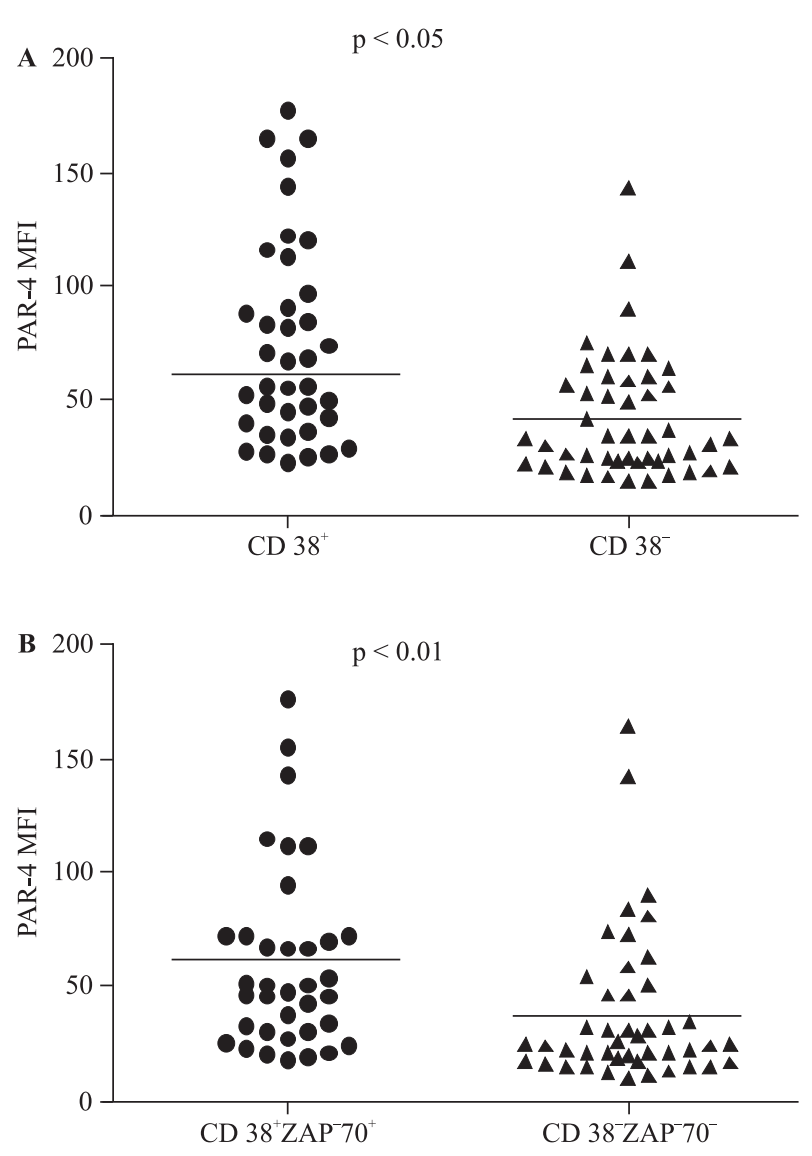

Figure 2. Median expression of PAR-4 protein in CD19+ $\mathrm{B}$ cells from $\mathrm{CD} 8^{+}$and $\mathrm{CD} 38^{-}$patients (A) as well as $\mathrm{CD}^{2} 8^{+} \mathrm{ZAP}^{-} 70^{+}$and $\mathrm{CD} 38^{+} \mathrm{ZAP}^{-} 70^{+}$patients $(\mathbf{B})$. $\mathrm{MFI}$ - mean fluorescence intensity

CD38 ${ }^{+} \mathrm{ZAP}-70^{+}$patients (median: $\left.65.38 \mathrm{MFI}\right)$ compared to CD38-ZAP-70- patients (median: 43.05 MFI) $(\mathrm{p}<0.01)$ (Figure 2B).

We found a direct correlation between LPL expression in $\mathrm{CD} 19^{+} / \mathrm{CD}^{+}$cells and PAR-4 protein expression $(\mathrm{R}=0.8 ; \mathrm{p}<0.000001)$. There was also a direct correlation between the expression of PAR-4 and the LPL/ADAM29 ratio $(\mathrm{R}=0.5 ; \mathrm{p}<0.00005)$.

Moreover, positive correlations between DAXX protein expression and LPL MFI $(\mathrm{R}=0.8 ; \mathrm{p}<$ $<0.000001$ ), as well as the LPL/ADAM29 MFI ratio $(\mathrm{R}=0.6 ; \mathrm{p}<0.0005)$ have been observed.

Likewise, there was a direct correlation between ZIPK protein expression and LPL MFI $(\mathrm{R}=0.6$; $\mathrm{p}<0.00005)$.

When compared, the advanced-stage (3-4 acc. Rai) patients showed a higher PAR-4 protein (56.73 MFI) expression than the early-stage ( 0 and 1-2 acc. Rai) patients (48.38 MFI), although the differences were not statistically significant $(\mathrm{p}=0.219)$. On the other hand, BCL-2 protein expression in B cells was lower in the advanced-stage patients

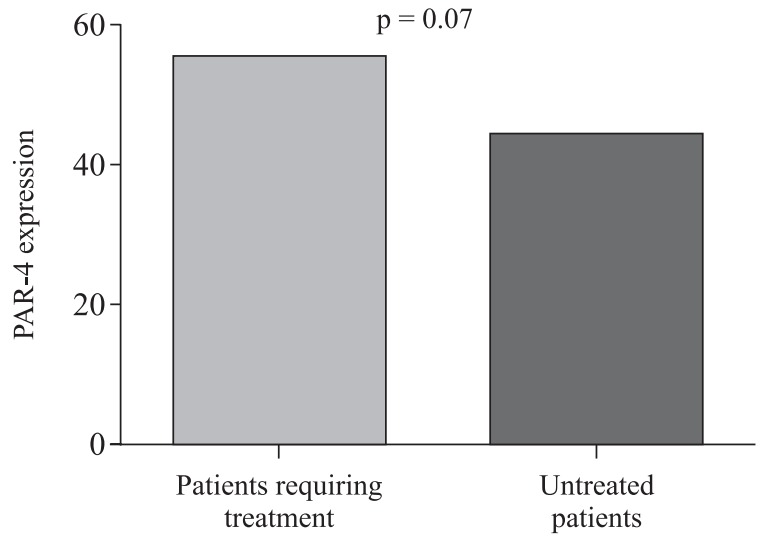

Figure 3. Median expression of PAR-4 protein in CD19+ $\mathrm{B}$ cells from patients requiring treatment and untreated patients

(65.19 MFI) compared to the early-stage ones (76.45 MFI) $(\mathrm{p}=0.147)$.

We found a higher expression of PAR-4 in the group of patients requiring treatment at diagnosis (median: 56.25 MFI) than in patients who did not (median: $45.71 \mathrm{MFI}$ ) (Figure 3). However, the difference was not statistically significant. Likewise, in patients who died after the measurement, the PAR-4 expression assessed at diagnosis was higher than in patients who survived (46.38 vs. $37.78 \mathrm{MFI}$; $=0.06$ ).

\section{Discussion}

PAR-4 (prostate apoptosis response-4) protein was originally found upregulated in prostate tumor cells undergoing apoptosis. Then its ubiquitous expression in different tissues of many species was demonstrated [12]. PAR-4 overexpression was further identified during apoptosis of a variety of cancer cells, such as renal cell carcinoma, malignant melanoma, colon carcinoma and pancreatic cancer $[6,9,21]$. There is also evidence that PAR-4 contributes to the induction of neuronal cell death [21] and it has been found as a proapoptotic protein upregulated both in normal and leukemic lymphocytes [9].

PAR-4 is involved in several protein-protein interactions and its binding partners include atypical protein kinase $\mathrm{C}(\mathrm{aPKC})$, the Wilm's tumor 1 (WT1), DAP-like kinase/ZIP kinase (DLK/ZIPK), THAP-domain protein 1 (THAP1), Dopamine D2 receptor and Akt $[4,12]$. Thus, PAR-4 is suggested to play a role both in extrinsic (ligand-mediated) and intrinsic (mitochondrially-mediated), as well as in nuclear apoptotic pathway, which might be of clinical significance [12].

The other protein assessed in our study, ZIP kinase (ZIPK, also known as DLK) together with 
DAPK, DAPK2, DRAK1 and DRAK2 constitute a family of related kinases that induce apoptosis upon overexpression [13, 14, 22]. ZIPK is involved in a nuclear pathway of apoptosis in PML oncogenic domains (PODs, nuclear domains that exist in all nucleated mammalian cells), where it binds and co-localizes with DAXX. Moreover, PAR-4, the other substrate of ZIPK, has been found to facilitate the association between DAXX and ZIPK.

The consequence of forming this complex is caspase activation and thus apoptosis induction from nuclei [13].

The third protein involved in the nuclear pathway of apoptosis mentioned above, DAXX (the death associated protein), has recently emerged as a multifunctional protein implicated in several important apoptotic pathways. Originally, DAXX was identified as a Fas-binding protein playing a role in the extrinsic pathway of apoptosis [23]. Subsequent experiments have demonstrated a range of different functions of DAXX both in the cytoplasmatic and nuclear localization. Most importantly, DAXX has been described as a promoter of apoptosis acting as an interaction partner of apoptosis signal-regulating kinase 1(ASK1), an apical kinase in the JNK and p38 pathway system. The phosphorylation of DAXX and PAR-4 by ZIP kinase in nuclear bodies (PODs) has been shown to induce the nuclear pathway of apoptosis $[13,15]$.

Since there is little data concerning the PAR-4 nuclear pathway of apoptosis in B-CLL, the aim of our study was to assess this protein expression in the B cells of CLL patients and examine the relationship between PAR-4 and other proteins involved in this apoptotic pathway, such as BCL-2, ZIP kinase and DAXX.

We found a positive relationship between PAR-4 and BCL-2 protein expression, and a positive correlation between PAR-4 and both DAXX and ZIPK protein expression, which confirms close relationships between these proteins in apoptosis induction. It has been previously observed that PAR-4 overexpression in nonhematopoietic cells results in inducing apoptosis by BCL-2 protein level downregulation $[9,10]$. Boehrer et al. found that PAR-4 mRNA was present in lymphocytes from most examined CLL samples, and PAR-4 protein was expressed in all CLL samples, while less frequently in immature lymphocytes of acute lymphoblastic leukemia (ALL). Furthermore, an inverse expressional pattern of PAR-4 and BCL-2 proteins was observed in ALL, whereas in CLL there was no obvious relationship between these proteins $[9,10]$. In our study, there was a direct correlation between PAR-4 and BCL-2. However, when compared, the advanced-stage patients showed a higher
PAR-4 protein expression and a lower BCL-2 protein expression than the early-stage patients (NS). This suggests that in CLL some other regulatory mechanisms must be involved in this interaction.

DAXX has been shown to be an important factor contributing to a good response to drug-induced apoptosis in lymphoma cells [24]. Boehrer et al. examined the enhancement of apoptosis by DAXX overexpression in neoplastic lymphocytes, which was proved to be caspase-dependent. They found that simultaneous overexpression of DAXX, PAR-4 and ZIPK proteins elicited a more than six-fold increase in apoptosis compared to control cells [15]. These results are in line with our results showing a positive correlation between PAR-4 and both DAXX and ZIPK protein expression, as well as between DAXX and ZIPK protein expression in CLL.

We observed a positive correlation between the expression of PAR-4 protein and the lactate dehydrogenase (LDH) serum concentration. There were also positive correlations between the expression of PAR-4 protein and such prognostic factors as the percentage of $\mathrm{CD} 38^{+}$cells, as well as LPL expression and the LPL/ADAM29 ratio. These results seem to confirm that PAR-4 assessment in malignancies might not only be prognostically important, but might also have important therapeutic implications. Discovery of agents inducing the PAR-4 expression may help in developing risk-adapted therapeutic strategies in CLL to enhance the effects of both chemotherapy and targeted therapies that involve different pathways of apoptosis [12].

\section{Acknowledgements}

This study was supported by research grant No. N N402 084234 from State Funds for Scientific Research.

\section{References}

1. Diaz-Meco MT, Moscat J. Akt regulation and lung cancer: a novel role and mechanism of action for the tumor suppressor Par-4.Cell Cycle. 2008;7:2817-2820.

2. Sells SF, Wood DP Jr, Barve SSJ et al. Commonality of the gene programs induced by effectors of apoptosis in androgendependent and -independent prostate cells. Cell Growth Differ. 1994;5:457-466.

3. Wang LH, Chen JY, Liou YJ et al. Association of missense variants of the PRKC, apoptosis, WT1, regulator (PAWR) gene with schizophrenia. Prog Neuropsychopharmacol Biol Psychiatry. 2008;32:870-875.

4. Affar el B, Luke MP, Gay F et al. Targeted ablation of Par-4 reveals a cell type-specific susceptibility to apoptosis-inducing agents. Cancer Res. 2006;66:3456-3462.

5. Sells SF, Han S-S, Muthukkumar S et al. Expression and function of the leucine zipper protein PAR-4 in apoptosis. Mol Cell Biol. 1997;17:3823-3832. 
6. Azmi AS, Wang Z, Burikhanov R et al. Critical role of prostate apoptosis response- 4 in determining the sensitivity of pancreatic cancer cells to small-molecule inhibitor-induced apoptosis. Mol Cancer Ther. 2008;7:2884-2893.

7. Xie J, Guo Q. Par-4 is a novel mediator of renal tubule cell death in models of ischemia-reperfusion injury. Am J Physiol Renal Physiol. 2007;292:F107-115.

8. Zhao Y, Burikhanov R, Qiu S et al. Cancer resistance in transgenic mice expressing the SAC module of Par-4. Cancer Res. 2007;67:9276-9285.

9. Boehrer S, Chow KU, Puccetti E et al. Deregulated expression of prostate apoptosis response gene-4 in less differentiated lymphocytes and inverse expressional patterns of par-4 and bcl-2 in acute lymphocytic leukemia. Hematol J. 2001;2:103-107.

10. Boehrer S, Chow KU, Beske F et al. In lymphatic cells par-4 sensitizes to apoptosis by downregulating bcl-2 and promoting disruption of mitochondrial membrane potential and caspase activation. Cancer Res. 2002;62:1768-1775 .

11. Gurumurthy S, Goswami A, Vasudevan KM, Rangnekar VM. Phosphorylation of Par-4 by protein kinase A is critical for apoptosis. Mol Cell Biol. 2005;25:1146-1161.

12. Mundle SD. Par-4: a common facilitator/enhancer of extrinsic and intrinsic pathways of apoptosis. Leuk Res. 2006;30:515-517.

13. Kawai T, Akira S, Reed JC. ZIP kinase triggers apoptosis from nuclear PML oncogenic domains. Mol Cell Biol. 2003;23:6174-6186.

14. Kawai T, Matsumoto M, Takeda K, Sanjo H, Akira S. ZIP kinase, a novel serine/threonine kinase which mediates apoptosis. Mol Cell Biol. 1998;18:1642-1651.

15. Boehrer S, Nowak D, Hochmuth S et al. Daxx overexpression in T-lymphoblastic Jurkat cells enhances caspase-dependent death receptor- and drug-induced apoptosis in distinct ways. Cell Signal. 2005;17:581-595.
16. Cheson BD, Bennett JM, Grever M et al. National Cancer Institute-sponsored Working Group guidelines for chronic lymphocytic leukemia: revised guidelines for diagnosis and treatment. Blood. 1996;87:4990-4997.

17. Rai KR, Sawitsky A, Cronkite EP, Chanana AD, Levy RN, Pasternack BS. Clinical staging of chronic lymphocytic leukemia. Blood. 1975;46:219-234.

18. Bojarska-Junak A, Giannopoulos K, Kowal M, Dmoszynska A, Rolinski J. Comparison of methods for determining zeta-chain associated protein-70 (ZAP-70) expression in patients with B-cell chronic lymphocytic leukemia (B-CLL). Cytometry B Clin Cytom. 2006;70:293-301.

19. Hus I, Podhorecka M, Bojarska-Junak A et al. The clinical significance of ZAP-70 and CD38 expression in B-cell chronic lymphocytic leukaemia. Ann Oncol. 2006;17:683-690.

20. Bojarska-Junak A, Hus I, Chocholska $S$ et al. BAFF and APRIL expression in B-cell chronic lymphocytic leukemia: correlation with biological and clinical features. Leuk Res. 2009;33:1319-1327.

21. Garcia-Cao I, Lafuente MJ, Criado LM, Diaz-Meco MT, Serrano M, Moscat J. Genetic inactivation of Par4 results in hyperactivation of NF-kappaB and impairment of JNK and p38. EMBO Rep. 2003;4:307-312.

22. Boosen M, Vetterkind S, Kubicek J, Scheidtmann KH, Illenberger S, Preuss U. Par-4 is an essential downstream target of DAP-like kinase (Dlk) in Dlk/Par-4-mediated apoptosis. Mol Biol Cell. 2009;20:4010-4020.

23. Yang X, Khosravi-Far R, Chang HY, Baltimore D. Daxx, a novel Fas-binding protein that activates JNK and apoptosis. Cell. 1997;89:1067-1076.

24. Chow KU, Nowak D, Boehrer S et al. Synergistic effects of chemotherapeutic drugs in lymphoma cells are associated with down-regulation of inhibitor of apoptosis proteins (IAPs), prostate-apoptosis-response-gene 4 (Par-4), deathassociated protein (Daxx) and with enforced caspase activation. Biochem Pharmacol. 2003;66:711-724.

Submitted: 20 June, 2010

Accepted after reviews: 21 November, 2010 